\title{
SOME NEW INEQUALITIES FOR MOTZKIN NUMBERS
}

\author{
TOMISLAV DOŠLIĆ
}

Abstract. We prove some inequalities which follow from the log-convexity of the sequence of Motzkin numbers $M_{n}$ and from the log-concavity of the sequence $\frac{M_{n}}{n !}$.

Mathematics subject classification (2000): 05A20, 26D99.

Keywords and phrases: Inequalities, Motzkin numbers, log-convexity, log-concavity, integer sequences.

\section{REFERENCES}

[1] M. Aigner, Motzkin numbers, European Journal of Combinatorics 19 (1998), 663-675.

[2] D. Callan, Notes on Motzkin and Schroeder Numbers, preprint.

[3] R. Donaghey and L. W. ShaPiro, Motzkin Numbers, Journal of Combinatorial Theory series A 23 (1977), 291-301.

[4] T. DošLIĆ, D. SVRTAN AND D. VelJAN, Secondary Structures, submitted.

[5] T. MotZKIN, Relation between hypersurface cross ratios, and a combinatorial formula for partitions of a polygon, for permanental preponderance and for non-associative products, Bulletin of American Mathematical Society 54 (1948), 352-360.

[6] R. Stanley, Enumerative Combinatorics II, Cambridge Univ. Press, Cambridge, 1999.

[7] D. Veluan, Combinatorial and Discrete Mathematics, Algoritam, Zagreb, 2001 (in Croatian).

[8] P. R. Stein And M. Waterman, On Some New Sequences Generalizing the Catalan and the Motzkin Numbers, Discr. Math. 26 (1978), 261-272. 